\title{
Curcumin attenuates cardiac fibrosis in spontaneously hypertensive rats through PPAR-y activation
}

\author{
Zhe MENG ${ }^{1, \#}$, Xin-hui $\mathrm{YU}^{2, \#}$, Jun CHEN ${ }^{1}$, Ling $\mathrm{LI}^{1,}$ *, Sheng $\mathrm{Ll}^{2, *}$ \\ ${ }^{1}$ Department of Cardiology, The First Affiliated Hospital of Zhengzhou University, Zhengzhou 450052, China; ${ }^{2}$ Department of Thoracic \\ Surgery, Taicang Affiliated Hospital of Soochow University, Taicang 215400, China
}

\begin{abstract}
Aim: To investigate the effects of curcumin (Cur) on cardiac fibrosis in spontaneously hypertensive rats (SHRs) and the mechanisms underlying the anti-fibrotic effect of Cur in rat cardiac fibroblasts (CFs) in vitro.

Methods: SHRs were orally treated with Cur $\left(100 \mathrm{mg} \cdot \mathrm{kg}^{-1} \cdot \mathrm{d}^{-1}\right)$ or Cur $\left(100 \mathrm{mg} \cdot \mathrm{kg}^{-1} \cdot \mathrm{d}^{-1}\right)$ plus the PPAR-y antagonist GW9662 $\left(1 \mathrm{mg} \cdot \mathrm{kg}^{-1} \cdot \mathrm{d}^{-1}\right)$ for 12 weeks. Cultured CFs were treated with angiotensin II (Ang II, $0.1 \mu \mathrm{mol} / \mathrm{L}$ ) in vitro. The expression of relevant proteins and mRNAs was analyzed using Western blotting and real-time PCR, respectively. The expression and activity of peroxisome proliferatoractivated receptor-y (PPAR-y) were detected using Western blotting and a DNA-binding assay, respectively.

Results: Treatment of SHRs with Cur significantly decreased systolic blood pressure, blood Ang II concentration, heart weight/body weight ratio and left ventricle weight/body weight ratio, with concurrently decreased expression of connective tissue growth factor (CTGF), plasminogen activator inhibitor (PAI)-1, collagen III (Col III) and fibronectin (FN), and increased expression and activity of PPAR-y in the left ventricle. Co-treatment with GW9662 partially abrogated the anti-fibrotic effects of Cur in SHRs. Pretreatment of CFs with Cur (5, 10, $20 \mu \mathrm{mol} / \mathrm{L})$ dose-dependently inhibited Ang II-induced expression of CTGF, PAI-1, Col III and FN, and increased the expression and binding activity of PPAR-y. Pretreatment with GW9662 partially reversed anti-fibrotic effects of Cur in vitro. Furthermore, pretreatment of CFs with Cur inhibited Ang II-induced expression of transforming growth factor- $\beta 1$ (TGF- $\beta 1$ ) and phosphorylation of Smad2/3, which were reversed by GW9662.

Conclusion: Cur attenuates cardiac fibrosis in SHRs and inhibits Ang Il-induced production of CTGF, PAI-1 and ECM in CFs in vitro. The crosstalk between PPAR- $\gamma$ and TGF- $\beta 1 /$ Smad2/3 signaling is involved in the anti-fibrotic and anti-proliferative effects of Cur.
\end{abstract}

Keywords: hypertension; cardiac fibrosis; curcumin; angiotensin II; GW9662; connective tissue growth factor; plasminogen activator inhibitor-1; PPAR-ү; Smad2/3

Acta Pharmacologica Sinica (2014) 35: 1247-1256; doi: 10.1038/aps.2014.63; published online 18 Aug 2014

\section{Introduction}

Cardiac fibrosis is a common pathological change that occurs as a results of both hypertension and myocardial infarction, and in the setting of valvular heart disease, which induces significant left ventricular hypertrophy. Therefore, it is regarded as a critical risk factor in determining clinical outcomes of cardiac diseases ${ }^{[1]}$. Cardiac fibroblasts (CFs) play a critical role in the progression of cardiac remodeling in the setting of hypertension by upregulating extracellular matrix (ECM) deposition in hearts ${ }^{[2]}$. Growing evidence has shown that the expression of angiotensin II (Ang II) is dramatically elevated in the set-

\footnotetext{
\# The first two authors contributed equally to this paper.

${ }^{*}$ To whom correspondence should be addressed.

E-mail liling63035@sina.com (Ling LI); lisheng_0512@126.com (Sheng LI)

Received 2014-01-01 Accepted 2014-06-05
}

ting of hypertension and myocardial infarction ${ }^{[3,4]}$. Increased Ang II causes cardiac fibrosis by inducing the proliferation and migration of CFs, over-apoptosis of cardiac myocardium, and over-deposition of ECM components such as collagen I (Col I), collagen III (Col III), and fibronectin $(\mathrm{FN})^{[4-6]}$.

Plasminogen activator inhibitor (PAI)-1, which belongs to the serine protease inhibitor family, amplifies the progression of fibrosis in various organs such as heart, lung, and kidney, by increasing ECM production, down-regulating ECM degeneration, and suppressing the activity of metalloproteinases ${ }^{[6,7]}$. The expression of PAI-1 is increased in both CFs and the myocardiums when Ang II is present ${ }^{[8,9]}$. The expression of connective tissue growth factor (CTGF) is increased in the left ventricle and arteries as a results of hypertension, atherosclerosis, and diabetes mellitus, in both animal models and patients $^{[10-12]}$. CTGF, a potential pro-fibrotic factor, plays an 
important role in accelerating the progression of cardiac and vascular fibrosis via the stimulation of proliferation of $\mathrm{CFs}$ and vascular smooth muscle cells ${ }^{[13,14]}$. It has been determined that the expression of CTGF is upregulated by Ang II, which suggests that CTGF may be a mediator of Ang II-induced cardiac fibrosis $^{[15]}$.

Peroxisome proliferator-activated receptor- $\gamma$ (PPAR- $\gamma$ ), a member of the nuclear hormone family, is involved in the regulation of a variety of physiological functions, such as glucose and lipid metabolism, inflammation, immune responses, and tissue fibrosis, as an activated PPAR- $\gamma$ binds to specific PPAR response elements (PPREs) and controls the expression of its target genes ${ }^{[8,16,17]}$. Growing evidence has indicated that upregulation of PPAR- $\gamma$ activity is an effective method to prevent cardiac fibrosis caused by hypertension, myocardial infarction or diabetes ${ }^{[11,14,18]}$. Recently, PPAR-ץ ligands have been shown to inhibit Ang II-induced over-production of ECM and proliferation of $\mathrm{CFs}^{[8]}$.

Curcumin (Cur), the main component of the yellow color of turmeric, is a natural polyphenol that has been used to treat a variety of diseases since ancient times ${ }^{[19]}$. Considerable evidence has indicated that Cur exhibits various beneficial physiological effects, such as anti-proliferative, anti-oxidative, anti-inflammatory, anti-carcinoma, and anti-infectious effects, by affecting a wide range of molecular targets including cytokines, growth factors, nuclear transcription factors, and intercellular adhesion molecules ${ }^{[20-23]}$. Cur has recently received attention for its protective effects on the cardiovascular system, such as its down-regulation of blood pressure, inhibition of the progression of atherosclerosis, and suppression of diabetic cardiomyopathy ${ }^{[24-26]}$. Cur also demonstrates a capacity to upregulate the activity of PPAR- $\gamma$, which inhibits both ischemia-induced inflammation in the brain and the activity of hepatic stellate cells caused by advanced glycation end-products ${ }^{[27,28]}$. However, whether Cur effectively inhibits Ang II-induced cardiac fibrosis via upregulation of PPAR- $\gamma$ has not been clearly demonstrated either in vivo or in vitro.

To better understand how Cur exerts its effects through Ang II, we studied the molecular mechanisms related to the inhibition of Ang II-induced expression of CTGF, PAI, and ECM by Cur in $\mathrm{CFs}$.

\section{Materials and methods Reagents}

Dulbecco's modified Eagle's medium (DMEM) was obtained from Gibco BRL (Carlsbad, CA, USA). Penicillin, curcumin, streptomycin, Tris, Ang II, GW9662, and 3-[4,5-dimethylthiazol-2-yl]-2,5-diphenyltetrazolium bromide (MTT) were purchased from Sigma Chemical Co (St Louis, MO, USA). Antibodies against PPAR- $\gamma$, TGF- $\beta 1$, Smad $2 / 3$, and phosphoSmad2/3 (p-Smad2/3) were purchased from Cell Signaling Technology (Beverly, MA, USA), and antibodies against CTGF, PAI-1, Col III, and FN were obtained from Abcam (Cambridge, UK). Transzol, fetal bovine serum (FBS), EasyScript Reverse Transcriptase, TransStrat Green Qpcr SuperMix, and a $\beta$-actin antibody were purchased from TransGen Bio- technology (Beijing, China).

\section{Animal experiments}

Eight-week-old male spontaneously hypertensive rats (SHRs) and age-matched male Wistar Kyoto (WKY) rats obtained from Shanghai SLAC Laboratory Animal Technique Corp were used. Normal WKY rats were chosen as the control group $(n=8)$. The SHRs were randomly divided into the following 3 groups ( $n=8$ each) for different treatments by oral gavage for 12 weeks: Cur $\left(100 \mathrm{mg} \cdot \mathrm{kg}^{-1} \cdot \mathrm{d}^{-1}\right)^{[29]}$, Cur (100 $\left.\mathrm{mg} \cdot \mathrm{kg}^{-1} \cdot \mathrm{d}^{-1}\right)+\mathrm{GW} 9662\left(10 \mathrm{mg} \cdot \mathrm{kg}^{-1} \cdot \mathrm{d}^{-1}\right)$, and saline control. All rats were housed under similar conditions with a 12-h light/ dark cycle at $23 \pm 1{ }^{\circ} \mathrm{C}$ and humidity $55 \% \pm 5 \%$. Rats had free access to an ordinary diet and water. Using the tail-cuff method, conscious rats had their systolic blood pressures (SBPs) measured. Body weight was recorded biweekly during the study period. At the end of the 12th week, rats were killed by intra-aortic administration of $10 \%$ potassium chloride and an excess amount of pentobarbital. Hearts were subsequently excised and weighed. One portion of the left ventricle was fixed in $4 \%$ formaldehyde solution and embedded in paraffin for collagen evaluation, and the remaining portion was snapfrozen in liquid nitrogen and stored at $-70^{\circ} \mathrm{C}$ for subsequent biochemical assays.

\section{Sirius red staining}

Collagen was evaluated using a Sirius Red assay. Left ventricle sections $(5 \mathrm{~mm})$ were hydrated and stained with Sirius Red ( $0.5 \%$ in saturated aqueous picric acid; Sigma). Ten fields in each region of the heart were selected randomly from four nonconsecutive serial sections, and collagen content was quantified as Sirius Red-positive areas using fluorescence confocal microscopy (Leica TCS SP2-AOB).

\section{Ang II concentration assay}

To measure the concentration of Ang II, blood samples of 4 groups were collected in chilled tubes containing ethylenediaminetetraacetic acid (EDTA, $20 \mathrm{~g} / \mathrm{L}$ ) pH 5.5, to inhibit any possible enzymatic generation and degradation of Ang II. Collected blood samples were placed into $1.5 \mathrm{~mL}$ microcentrifuge tubes and centrifuged at $3000 \times \mathrm{g}$ and $4{ }^{\circ} \mathrm{C}$ for 15 min. The plasma samples were then stored at $-20^{\circ} \mathrm{C}$ for subsequent analysis. Determination of Ang II was performed using a commercial enzyme-linked competitive immunoassay kit (Elabs, Gothenburg, Sweden), following the manufacturer's protocol.

\section{Cell culture}

All animal procedures and our studies followed the Guide for the Care and Use of Laboratory Animals published by the US National Institutes of Health (NIH Publication No 85-23, revised 1996). Sprague-Dawley rats (1-to-2 d old) were obtained from the Laboratory Animal Institute in the School of Medicine at Zhengzhou University. Collagenase and trypsin digestion were used to obtain the CFs from the rats' ventricles using a previously published method ${ }^{[30]}$. CFs were cultured 
in DMEM with 15\% FBS, $100 \mathrm{U} / \mathrm{mL}$ penicillin and $100 \mu \mathrm{g} / \mathrm{mL}$ streptomycin in a humidified atmosphere of $5 \% \mathrm{CO}_{2}$ and $95 \%$ air at $37^{\circ} \mathrm{C}$. CFs were identified by examination of morphology and immunocytochemistry. Cells within 4 passages were used for the experiments. When the cells reached $80 \%-90 \%$ confluence, the medium was replaced with serum-free medium and cells were cultured for $24 \mathrm{~h}$ before conducting the experiments.

\section{Cell proliferation assay}

Cell proliferation was determined using the MTT reduction assay. CFs were seeded at a density of 8000 cells/well, in 96-well plates. After each treatment, the medium was removed, and cells were incubated with MTT $(5 \mathrm{mg} / \mathrm{mL})$ for $4 \mathrm{~h}$ at $37^{\circ} \mathrm{C}$. The resulting dark blue formazan crystals that formed inside the intact cells were solubilized with DMSO, and absorbance was measured at $490 \mathrm{~nm}$ on a microplate spectrophotometer (POLARstar OPTIMA, BMG LABTECH, Offenburg, Germany).

\section{Real-time reverse transcription-PCR}

Total RNA was extracted using TransZol reagent, and DNA was removed using a DNA-free kit (Ambion, Austin TX, USA). The quality of the mRNA was checked by performing denaturing agarose gel electrophoresis containing 1.5\% formaldehyde. The total RNA concentration and purity were determined by UV-Vis spectroscopy with the Bio-Rad SmartSpec 3000 system (Bio-Rad, Hercules, CA, USA). To synthesize cDNA, $1 \mu \mathrm{g}$ of total RNA was included in a $20 \mu \mathrm{L}$ reaction utilizing oligo(dT)18 Primer and TransScript Reverse Transcriptase (TransGen Biotech, Beijing). Primers for rat PPAR-ү, CTGF, PAI-1, collagen III, fibronectin, and GAPDH were chosen using Beacon designer v4.0 (Premier Biosoft, CA, USA). GAPDH was used as an endogenous control. The mRNA levels of PPAR- $\gamma$, CTGF, PAI-1, collagen III, fibronectin, and GAPDH were measured by real-time PCR with the ABI PRISM 7000 sequence detection PCR system (Applied Biosystems, Foster City, CA, USA). One melting peak confirmed the presence of a single product. The results were expressed as fold differences relative to the level of $\beta$-actin using the $2^{-\Delta \Delta C T}$ method.

\section{Western blot analysis}

Either CFs or myocardial tissue were lysed with $200 \mu \mathrm{L}$ of ice-cold lysis buffer ( $\mathrm{pH} 7.4)(50 \mathrm{mmol} / \mathrm{L}$ HEPES, $5 \mathrm{mmol} / \mathrm{L}$ EDTA, $100 \mathrm{mmol} / \mathrm{L} \mathrm{NaCl}, 1 \%$ Triton X-100, protease inhibitor cocktail; Roche, Mannheim, Germany) in the presence of phosphatase inhibitors $(50 \mathrm{mmol} / \mathrm{L}$ sodium fluoride, $1 \mathrm{mmol} / \mathrm{L}$ sodium orthovanadate, $10 \mathrm{mmol} / \mathrm{L}$ sodium pyrophosphate, 1 nmol/L microcystin). The activated PPAR- $\gamma$ protein, located in the nucleus, was extracted using a Pierce NE-PER kit (Pierce, Rockford, IL, USA). Protein concentrations were identified using a BCA protein assay kit. Samples underwent 10\% SDS-PAGE and were subsequently transferred onto a polyvinylidene difluoride membrane in a semi-dry system (BioRad), which was blocked with $5 \%$ fat-free milk in TBST buffer
(20 mmol/L Tris- $\mathrm{HCl}, 137 \mathrm{mmol} / \mathrm{L} \mathrm{NaCl}$, and $0.1 \%$ Tween 20). Samples were then incubated overnight with primary antibodies against PPAR- $\gamma$ (1:400), CTGF (1:500), PAI-1 (1:400), Col III (1: 1000), FN (1:500), $\beta$-actin (1:1000), and histone (1:500) in TBST buffer before being washed and incubated with secondary antibodies (1:5000) for $100 \mathrm{~min}$. The optical densities of the bands were quantified using a Gel-Pro Analyzer v4.0 (Media Cybernetics, Rockville, MD, USA). $\beta$-Actin was used as the endogenous control. Data were normalized against those of the corresponding $\beta$-actin. The results were expressed as fold differences compared with the control (Table 1).

Table 1. Primers used for real-time PCR analysis.

\begin{tabular}{cl}
\hline Gene & Oligonucleotide primer sequences $\left(5^{\prime}-3^{\prime}\right)$ \\
\hline PPAR-y & Forward: TGGAGCCTAAGTTTGAGTTTG \\
& Reverse: ATCTTCTGGAGCACCTTGG \\
CTGF & Forward: AAGAAGACTCAGCCAGACC \\
& Reverse: TGGAAAGAAGCTGAGGAAGG \\
PAl-1 & Forward: CCTCCTCATCCTGCCTAAGTTC \\
& Reverse: GCCGCTCTCGTTCACCTC \\
Col III & Forward: AGATGCTGGTGCTGAGAAG \\
& Reverse: TGGAAAGAAGCTGAGGAAGG \\
FN & Forward: GTGAAGAACGAGGAGGATGTG \\
& Reverse: GTGATGGCGGATGATGTAGC \\
GAPDH & Forward: GCCTTCTCCATGGTGGTGAA \\
& Reverse: GGTCGGTGTGAACGGATTTG \\
\hline
\end{tabular}

\section{DNA-binding assay}

Nuclear proteins were extracted using a Pierce NE-PER kit. PPAR- $\gamma$ DNA-binding activity was determined by an ELISAbased method using a PPAR- $\gamma$ transcription assay kit (Cayman Chemical, USA). As described previously, $10 \mathrm{mg}$ nuclear protein was added to a 96-well plate, which was prepared with PPAR specific double-stranded DNA containing the sequence for a peroxisome proliferator-response element (PPRE), and subsequently incubated for $12 \mathrm{~h}$ at $4{ }^{\circ} \mathrm{C}$. A specific PPAR- $\gamma$ antibody was used to detect bound PPAR- $\gamma$. To enable colorimetric reading at $450 \mathrm{~nm}$, there was a subsequent addition of a horseradish peroxidase-conjugated secondary antibody.

\section{Statistical analysis}

Results are expressed as the mean \pm SEM of three experiments. Differences between groups were assessed by one-way ANOVA followed by post hoc tests. Statistical tests were performed using SPSS 17.0 software (SPSS Inc, Chicago, IL, USA). A value of $P<0.05$ was considered statistically significant.

\section{Results}

Effect of Cur on SBP, body weight, heart weight, left ventricle weight, and Ang II concentration in SHRs

After 12 weeks of treatment, SBPs in the SHRs without Cur treatment was significantly higher than that in WKY rats, 
and the ratios of heart weight/body weight and left ventricle weight/body weight were also increased in SHRs. Meanwhile, the concentration of Ang II was significantly elevated in SHRs. Treatment with Cur $\left(100 \mathrm{mg} \cdot \mathrm{kg}^{-1} \cdot \mathrm{d}^{-1}\right)$ significantly inhibited the elevation of SBPs and the ratios of heart weight/ body weight and left ventricle weight/body weight, as well as the concentration of Ang II, in SHRs. Treating SHRs with a combination of GW9662 and Cur reversed this attenuation. These results suggest that Cur suppresses cardiac remolding in SHRs, which is related to PPAR- $\gamma$ activation. However, there were no differences in body weight among the different groups (Table 2).

Table 2. Effect of Cur on SBP, body weight, heart weight, and left ventricle weight in SHRs. Data are presented as mean \pm SEM of 8 rats in each group. ${ }^{\mathrm{c}} P<0.01$ vs WKY. ${ }^{\mathrm{f}} P<0.01$ vs SHRs. ${ }^{\mathrm{i}} P<0.01$ vs SHRs + Cur.

\begin{tabular}{lcccc}
\hline Parameter & WKY & SHRs & SHRs+Cur & $\begin{array}{c}\text { SHRs+Cur+ } \\
\text { GW9662 }\end{array}$ \\
\hline SBP (mmHg) & $127 \pm 3$ & $173 \pm 4^{\circ}$ & $152 \pm 4^{\dagger}$ & $165 \pm 6^{i}$ \\
Body weight (g) & $374 \pm 5$ & $372 \pm 6$ & $377 \pm 7$ & $373 \pm 6$ \\
HW/BW (mg/g) & $3.17 \pm 0.07$ & $3.41 \pm 0.08^{\mathrm{c}}$ & $3.27 \pm 0.05^{\mathrm{f}}$ & $3.37 \pm 0.08^{\mathrm{i}}$ \\
LVW/BW (mg/g) & $2.15 \pm 0.07$ & $2.29 \pm 0.07^{\mathrm{C}}$ & $2.20 \pm 0.07^{\dagger}$ & $2.25 \pm 0.07^{\mathrm{i}}$ \\
Ang II (pg/mL) & $118.6 \pm 15.3$ & $151.4 \pm 20.3^{\mathrm{c}}$ & $143.3 \pm 13.4^{\dagger}$ & $148.5 \pm 15.5^{\mathrm{i}}$ \\
\hline
\end{tabular}

WKY, Wistar Kyoto rats; SHRs, spontaneously hypertensive rats; Cur, curcumin; SBP, systolic blood pressure; BW, body weight; HW, heart weight; LVW, left ventricle weight; Ang II: angiotensin II.

Effect of Cur on collagen deposition in the left ventricles of SHRs The collagen content of the left ventricle was much higher in SHRs compared with WKY rats, as determined by Sirius Red staining. Treating SHRs with Cur $\left(100 \mathrm{mg} \cdot \mathrm{kg}^{-1} \cdot \mathrm{d}^{-1}\right)$ partially suppressed collagen deposition in the left ventricle, an effect that was inhibited by treatment with GW9662 in SHRs (Figure 1). These results indicate that activation of PPAR- $\gamma$ may be involved in the inhibitory effect of Cur on collagen deposition in the left ventricle of SHRs.
Effect of Cur on the expression of CTGF, PAI-1, Col III, and FN in the left ventricles of SHRs

The expression of CTGF and PAI-1 in the left ventricles was significantly higher in SHRs compared with WKY rats at the level of both mRNA and protein, but SHRs treated with Cur had levels only slightly higher than the WKY rats (Figure 2A and $2 \mathrm{C}$ ). The relative differences in mRNA and protein levels for Col III and FN were similar to CTGF and PAI-1 across the groups (Figure 2B and 2D). Treating SHRs with GW9662 reversed Cur's inhibitory effect on the expression of CTGF, PAI-1, Col III, and FN in the left ventricles of SHRs. These results suggest that PPAR- $\gamma$ activation may be involved in the procession of Cur's inhibitory effect on the expression of CTGF, PAI-1, Col III, and FN in the left ventricles of SHRs.

\section{Effect of Cur on the expression of PPAR-y in the left ventricles of SHRs}

Protein expression of PPAR-y in the left ventricles of SHRs was significantly lower in SHRs, compared with WKY rats, but pretreatment with Cur resulted in higher PPAR-ץ levels, reducing the difference between the two rat types. Treating SHRs with GW9662 significantly reversed Cur's upregulation of the protein expression of PPAR-Y (Figure 3A). A similar trend was observed for PPAR- $\gamma$ mRNA levels between these groups (Figure 3B).

\section{Effect of Cur on the activation of PPAR-y in Ang II-induced CFs}

Once activated, PPAR- $\gamma$ is translocated from the cytosol into the nucleus. Therefore, to observe the effect of Cur on the activation of PPAR- $\gamma$, we extracted nuclear protein from Ang IIinduced CFs. According to Western blot results, stimulation of CFs with Ang II (0.1 $\mu \mathrm{mol} / \mathrm{L})$ caused a significant decrease in PPAR- $\gamma$ expression; however treatment with Cur attenuated this effect in a concentration-dependent manner (Figure 4A). Additionally, the binding activity of PPAR- $\gamma$ in Ang IIinduced CFs was increased by Cur in a dose-dependent manner (Figure 4B).

Effect of Cur on the expression of CTGF, PAI-1, Col III, and FN in Ang II-induced CFs

Stimulation of CFs with Ang II $(0.1 \mu \mathrm{mol} / \mathrm{L})$ caused a signifi-
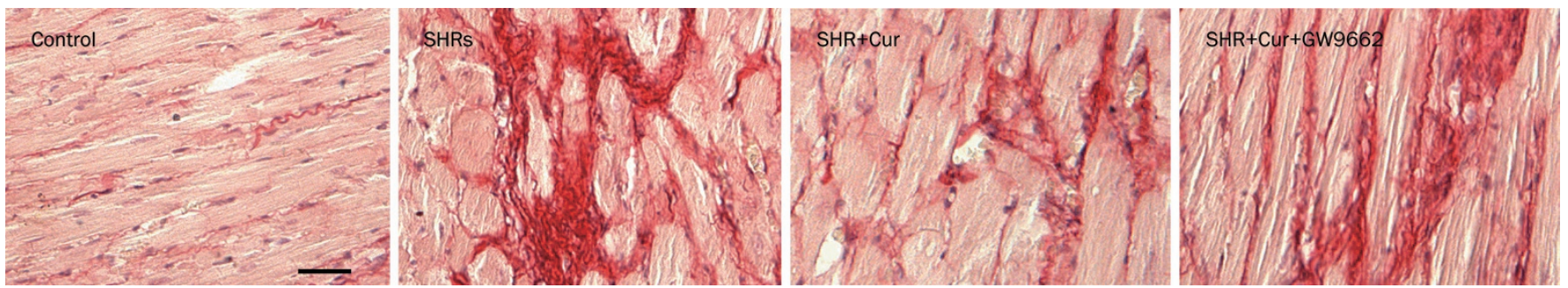

Figure 1. Cur decreased collagen deposition in the left ventricles of SHRs. WKY rats were used as controls, and SHRs were treated with saline, Cur (100 $\left.\mathrm{mg} \cdot \mathrm{kg}^{-1} \cdot \mathrm{d}^{-1}\right)$, or Cur $\left(100 \mathrm{mg} \cdot \mathrm{kg}^{-1} \cdot \mathrm{d}^{-1}\right)+\mathrm{GW} 9662\left(10 \mathrm{mg} \cdot \mathrm{kg}^{-1} \cdot \mathrm{d}^{-1}\right)$ by oral gavage for 12 weeks. Sirius Red staining was used to analyze the levels of collagen deposition in the left ventricles of WKY or SHRs ( $n=8$ rats, each group). Scale bar: $100 \mu \mathrm{m}$. WKY: Wistar Kyoto rats; SHRs: spontaneously hypertensive rats. 
A
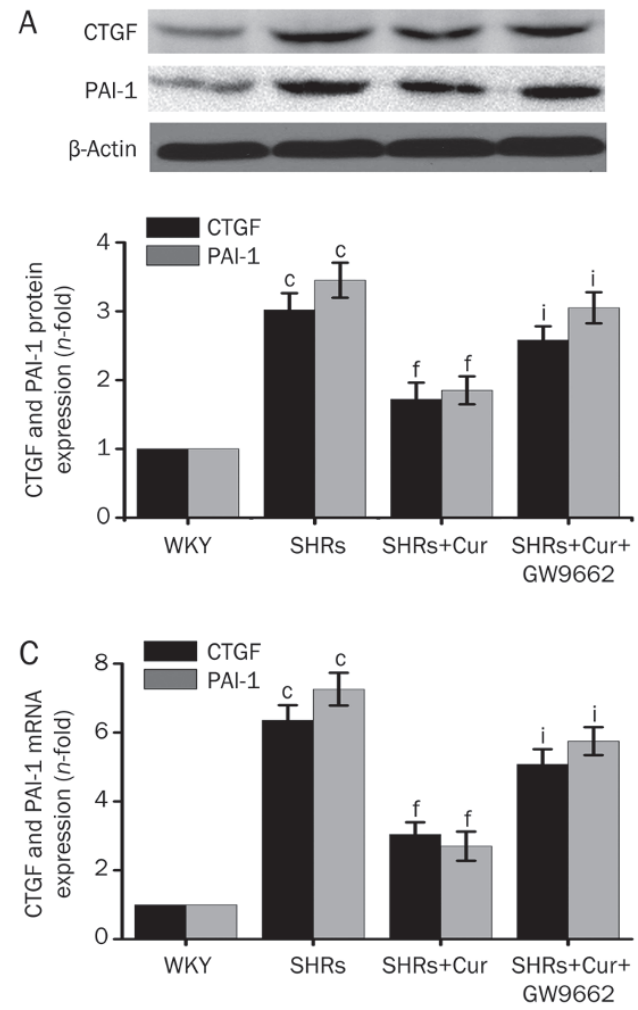

B
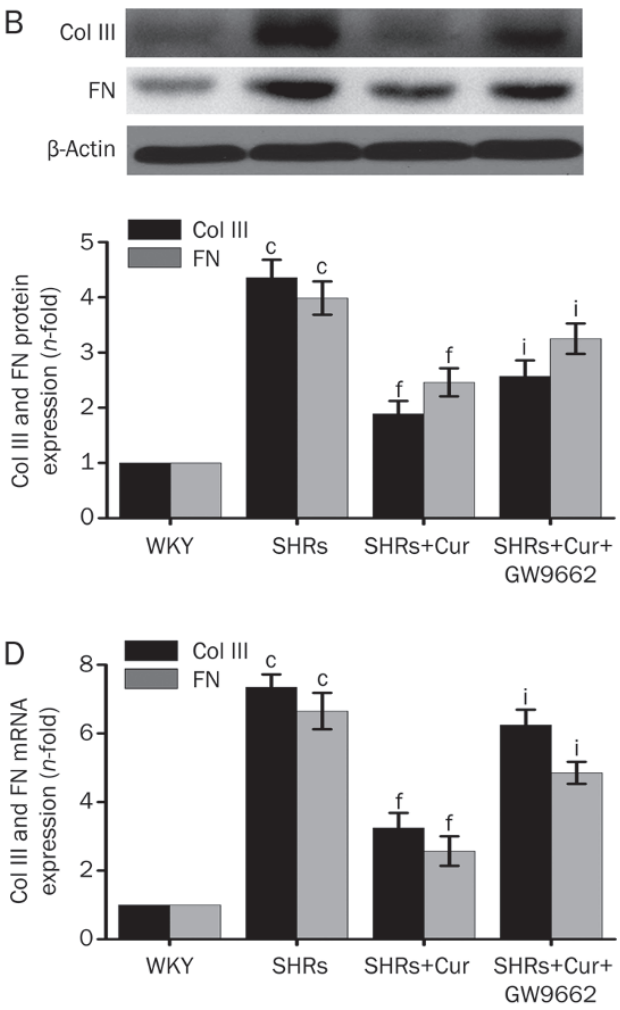

Figure 2. Cur downregulated mRNA and protein expression of CTGF, PAI-1, Col III, and PAI-1 in SHRs. WKY rats were used as controls, and SHRs were treated with saline, Cur $\left(100 \mathrm{mg} \cdot \mathrm{kg}^{-1} \cdot \mathrm{d}^{-1}\right)$, or Cur $\left(100 \mathrm{mg} \cdot \mathrm{kg}^{-1} \cdot \mathrm{d}^{-1}\right)+\mathrm{GW} 9662\left(10 \mathrm{mg} \cdot \mathrm{kg}^{-1} \cdot \mathrm{d}^{-1}\right)$ by oral gavage for 12 weeks. Western blotting was used to measure the expression of CTGF (A), PAI-1 (A), FN (B), and Col III (B). The mRNA expression of CTGF (C), PAI-1 (C), FN (D), and Col III (D) was detected by real-time PCR ( $n=8$ rats, each group). Data are presented as the mean \pm SEM of three independent experiments. ${ }^{\circ} P<0.01$ vs WKY. ${ }^{f} P<0.01$ vs SHRs. i $P<0.01$ vs SHRs+Cur.

A
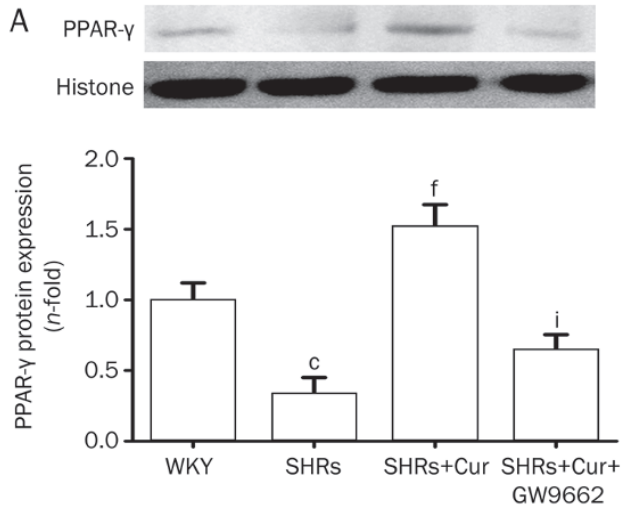

B

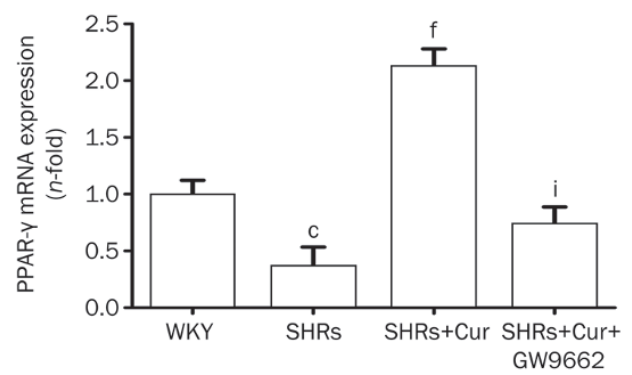

Figure 3. Cur increased the activation of PPAR-y in SHRs. WKY rats were used as controls, and SHRs were treated with saline, Cur $\left(100 \mathrm{mg}^{-1} \mathrm{~g}^{-1} \cdot \mathrm{d}^{-1}\right)$, or Cur $\left(100 \mathrm{mg} \cdot \mathrm{kg}^{-1} \cdot \mathrm{d}^{-1}\right)+\mathrm{GW} 9662\left(10 \mathrm{mg} \cdot \mathrm{kg}^{-1} \cdot \mathrm{d}^{-1}\right)$ by oral gavage for 12 weeks. The expression and activation of PPAR-y in the left ventricle of rats in each group was analyzed by Western blotting $(A)$ and a DNA-binding assay using a PPAR-y transcription assay kit (B). Data are presented as the mean \pm SEM of three independent experiments. ${ }^{C} P<0.01$ vs WKY. ${ }^{f} P<0.01$ vs SHRs. ${ }^{i} P<0.01$ vs SHRs + Cur.

cant increase in CTGF, Col I, Col III, and FN production, at the protein level. Cur inhibited Ang II-induced expression of CTGF, Col I, Col III, and FN in a concentration-dependent manner in CFs (Figure 5A, 5B). Cur also suppressed an Ang II-induced increase in mRNA expression of CTGF, Col I,
Col III, and FN, an effect that was also concentration-dependent (Figure 5C, 5D). However, after treating CFs with the PPAR-y antagonist GW9662, suppression of CTGF, Col I, Col III, and FN by Cur was not observed (Figure 4). 

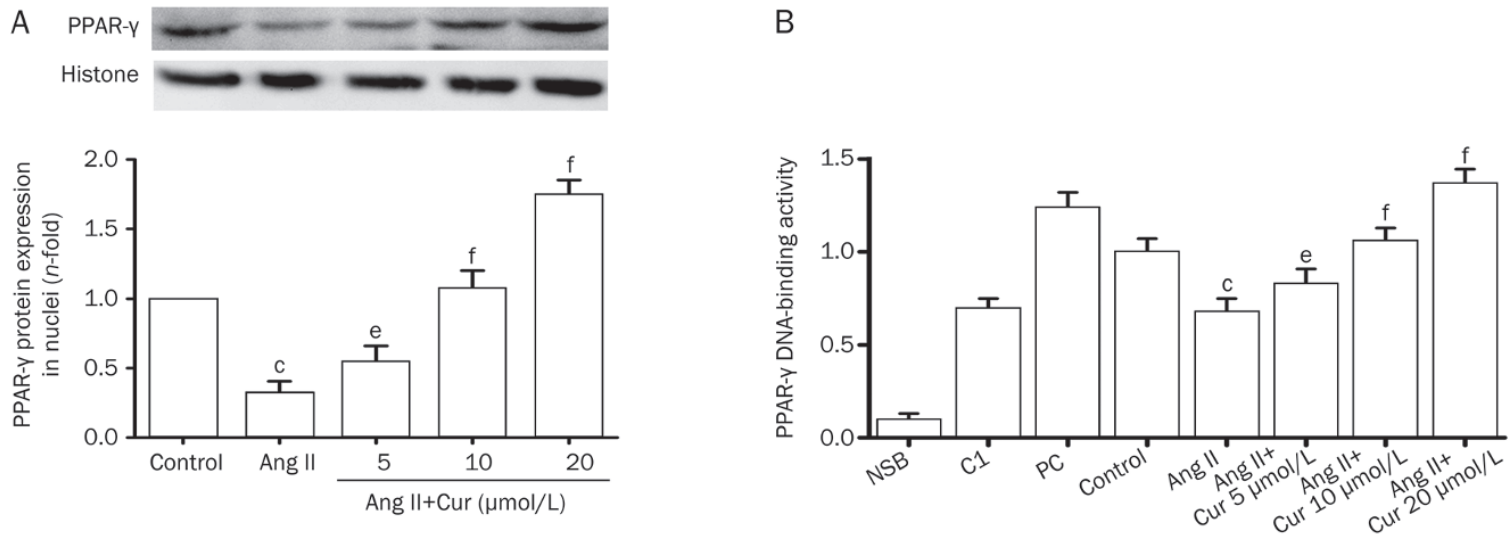

Figure 4. Cur increased the activation of PPAR-y in Ang Il-induced CFs. The cells were pretreated with Cur (5, 10, and 20 mol/L) for $1 \mathrm{~h}$, and subsequently stimulated with Ang II $(0.1 \mu \mathrm{mol} / \mathrm{L})$ for $24 \mathrm{~h}$. Nuclear protein was extracted, and the expression of PPAR-y was subsequently measured using Western blotting (A). PPAR- $y$ activation was detected by DNA-binding assay using a PPAR-y transcription assay kit (B). NSB: nonspecific binding; C1: competitor; PC: positive control. Data are presented as the mean \pm SEM of three independent experiments. ${ }^{\mathrm{C}} P<0.01 \mathrm{vs}$ control. ${ }^{\mathrm{e}} P<0.05,{ }^{\mathrm{f}} P<0.01 \mathrm{vS}$ Ang II. ' $P<0.01$ vs Ang II+Cur $20 \mu \mathrm{mol} / \mathrm{L}$.

A
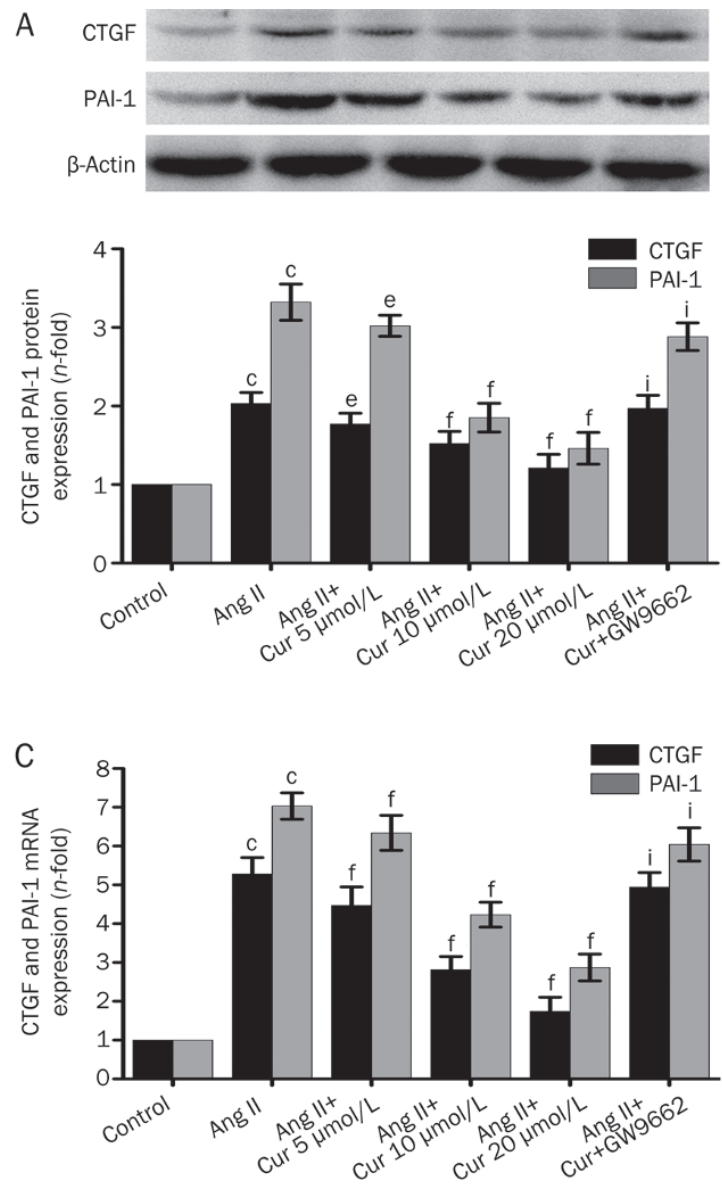

B
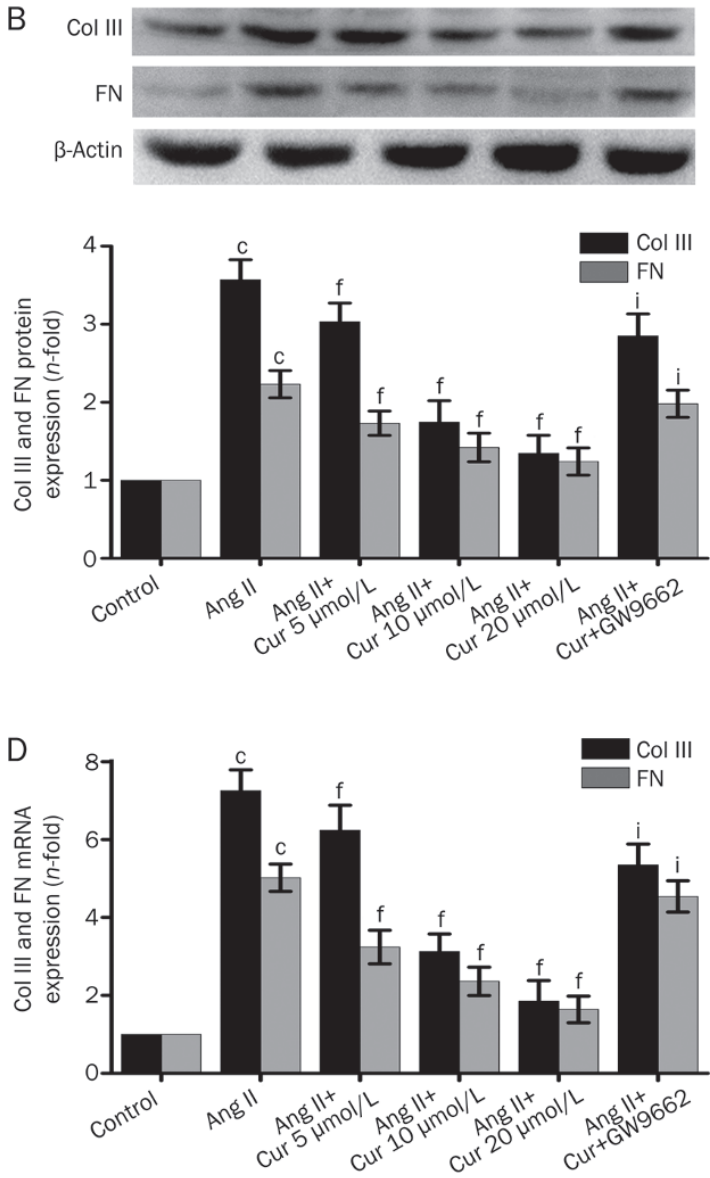

Figure 5. Cur inhibited Ang II-induced expression of CTGF, PAI-1, Col III, and FN in CFs. The cells were pretreated with Cur (5, 10, and $20 \mu \mathrm{mol} / \mathrm{L}$ ) with or without GW9662 $(5 \mu \mathrm{mol} / \mathrm{L})$ for $1 \mathrm{~h}$, and then subsequently stimulated with Ang II $(0.1 \mu \mathrm{mol} / \mathrm{L})$ for $24 \mathrm{~h}$. Western blotting was used to measure the protein expression of CTGF (A), PAI-1 (A), Col III (B) and FN (B). Some cells were pretreated with Cur (5, 10, and $20 \mu \mathrm{mol} / \mathrm{L})$, with or without GW9662 (5 $\mu \mathrm{mol} / \mathrm{L})$, for $1 \mathrm{~h}$, and subsequently stimulated with Ang II (0.1 $\mu \mathrm{mol} / \mathrm{L})$ for $9 \mathrm{~h}$. The mRNA expression of CTGF (C), PAI-1 (C), Col III (D), and FN (D) was analyzed by real-time PCR. Data are presented as the mean \pm SEM of three independent experiments. ${ }^{\mathrm{c}} P<0.01 \mathrm{vs}$ control. ${ }^{\mathrm{e}} P<0.05,{ }^{\mathrm{f}} P<0.01$ vs Ang II. 'P $<0.01$ vs Ang II+Cur $20 \mu \mathrm{mol} / \mathrm{L}$. 


\section{Effect of Cur on cell proliferation in Ang II-induced CFs}

Over-proliferation of CFs plays an important role in the progression of cardiac fibrosis. Therefore, we measured the effect of Cur on cell proliferation in Ang II-induced CFs. As shown in Figure 3, Ang II induced a remarkable increase in the cell viability. However, pretreating cells with Cur attenuated Ang II-induced CFs proliferation in a concentration-dependent manner. Furthermore, pretreatment with GW9662 in CFs suppressed Cur's inhibitory effect on Ang II-induced proliferation, suggesting a PPAR- $\gamma$-dependent mechanism (Figure 5).

\section{Effect of Cur on the activation of a TGF- $\beta 1 / \mathrm{Smad} 2 / 3$ signaling pathway in Ang II-induced CFs}

The TGF- $\beta 1 / S m a d 2 / 3$ signaling pathway has been shown to be a major mediator in regulating CTGF and PAI expression. As Cur mediates the activation of TGF- $\beta 1 / \mathrm{Smad} 2 / 3$ signaling, we investigated whether TGF- $\beta 1 / \mathrm{Smad} 2 / 3$ signaling is involved in Cur's inhibitory effect on CTGF, PAI, and ECM synthesis. As shown in Figure 6A, after stimulation with Ang II for $24 \mathrm{~h}$, protein expression of TGF- $\beta 1$ was increased significantly, and pretreatment with Cur $(5,10$, and $20 \mu \mathrm{mol} / \mathrm{L}$ ) attenuated this effect (Figure 7A). Moreover, the inhibitory effect of Cur was detected on the phosphorylation of Smad2/3 in CFs: this is TGF- $\beta 1$ 's main downstream target. Although Cur suppressed the Ang II-induced change in TGF$\beta 1$ production and the phosphorylation of Smad2/3 in CFs, it did not affect the total expression of Smad2/3 (Figure 7B). Moreover, treating CFs with GW9662 significantly reversed Cur's inhibitory effect on the activation of TGF- $\beta 1 / \mathrm{Smad} 2 / 3$ signaling (Figure 7). These results suggest that the mechanism by which Cur inhibits the production of pro-fibrotic factors and ECM in CFs partially involves the activation of TGF$\beta 1 /$ Smad2/3 signaling, which may be partially regulated by PPAR-ץ activation.

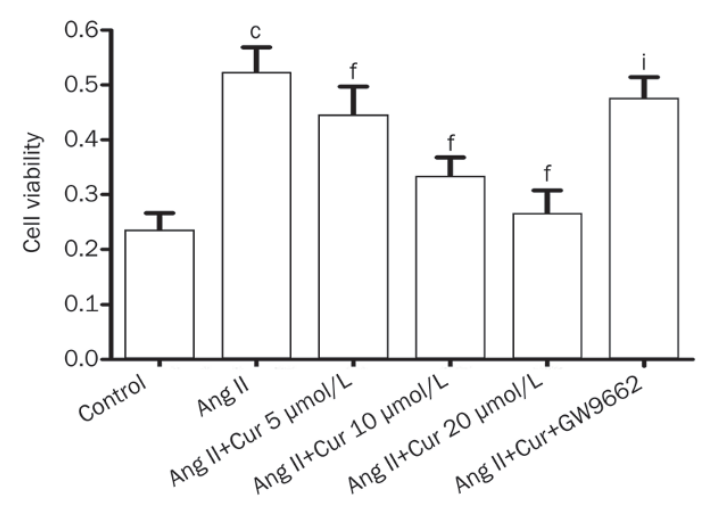

Figure 6. Cur suppressed Ang Il-induced proliferation of CFs. CFs were pretreated with Cur $(5,10$, and $20 \mu \mathrm{mol} / \mathrm{L})$ with or without GW9662 (5 $\mu \mathrm{mol} / \mathrm{L})$, for $1 \mathrm{~h}$, and subsequently stimulated with Ang II $(0.1 \mu \mathrm{mol} / \mathrm{L})$ for $24 \mathrm{~h}$. The proliferation of CFs was detected by MTT assay. Data are presented as the mean \pm SEM of three independent experiments. ${ }^{\mathrm{C}} P<0.01$ vs control. ${ }^{\mathrm{f}} P<0.01$ vs Ang II. ${ }^{\mathrm{i}} \mathrm{P}<0.01$ vs Ang $\mathrm{II}+\mathrm{Cur} 20 \mu \mathrm{mol} / \mathrm{L}$.
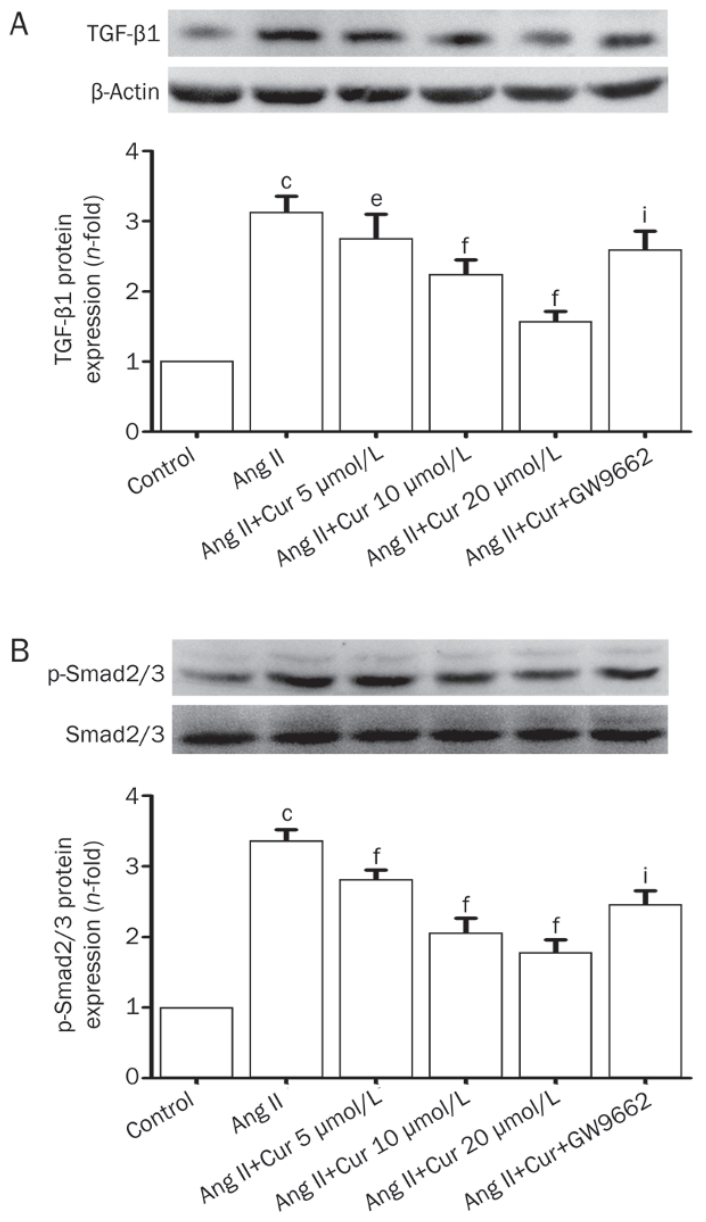

Figure 7. Cur inhibited Ang II-induced activation of TGF- $\beta 1 / \mathrm{Smad} 2 / 3$ signaling pathway in CFs. The cells were pretreated with Cur $(5,10$, and $20 \mu \mathrm{mol} / \mathrm{L})$ and subsequently stimulated with Ang $\mathrm{II}(0.1 \mu \mathrm{mol} / \mathrm{L})$ for another $24 \mathrm{~h}$. A Western blotting assay was used to measure the expression of TGF- $\beta 1$ (A) and the phosphorylation of Smad2/3 (B). Data are presented as the mean \pm SEM of three independent experiments. ${ }^{\mathrm{c}} P<0.01$ vs control. ${ }^{\mathrm{f}} P<0.01$ vs Ang II. ${ }^{\mathrm{i}} P<0.01$ vs Ang II+Cur $20 \mu \mathrm{mol} / \mathrm{L}$.

\section{Discussion}

One of the critical causes of cardiac fibrosis is hypertension. In the present study, we demonstrated that Cur significantly suppresses Ang II-induced CTGF and ECM production both in vivo and in vitro. Furthermore, we documented that Cur's inhibitory effect is partially dependent on the activation of PPAR- $\gamma$. These results expand the understanding of the key role of PPAR- $\gamma$ in cardiac fibrosis and provide novel experimental evidence of Cur's protective effect against hypertension-induced cardiac fibrosis.

The concentration of Ang II in the serum is remarkably elevated in the setting of hypertension, and causes cardiac fibrosis via the induction of CFs proliferation, excessive apoptosis of the myocardium, and excessive deposition of ECM. Col I, Col III, and FN constitute the main components of the ECM in cardiac fibrosis ${ }^{[6,8,30]}$. Growing evidence has indicated that 
the activity of PPAR- $\gamma$ is involved in the regulation of ECM metabolism in the heart, via the inhibition of ECM production, the upregulation of matrix MMPs activtity, and the suppression of pro-fibrotic factors ${ }^{[8,29,31]}$. More importantly, Cur inhibits pressure-overload-induced ECM production in the rabbit left ventricle and PDGF-induced expression of collagen in vascular smooth muscle cells (VSMCs) ${ }^{[32,33]}$. In this study, we determined that Cur inhibited Ang II-induced expression of $\mathrm{Col}$ III and FN in a dose-dependent manner, and this effect was partially reversed by the PPAR- $\gamma$ antagonist, GW9662. This study suggests that Cur effectively suppresses Ang IIinduced $\mathrm{ECM}$ production in $\mathrm{CFs}$, a process that is partially mediated by PPAR- $\gamma$.

CTGF is a potential factor in the over-proliferation of various connective tissue cells such as CFs, skin fibroblasts, and VSMCs, in the setting of systemic sclerosis, liver cirrhosis, and atherosclerosis ${ }^{[14,34]}$. Several studies have shown that CTGF, which is expressed by both cardiac fibroblasts and myocardium, promotes the progression of cardiac fibrosis ${ }^{[1,35]}$. Ang II induces over-production of CTGF in CFs and VSMCs at both the mRNA and protein levels; this is accomplished by suppressing the expression and activity of PPAR- $\gamma^{[14,36]}$. Additionally, it has been noted previously that the expression of CTGF in the wall of aorta of SHRs and Ang II-induced rats is controlled by PPAR- $\gamma^{[37]}$. More importantly, the activation of PPAR- $\gamma$, which is caused by ligands such as rosiglitazone and $15 \mathrm{~d}-\mathrm{PGJ}_{2}$, suppressed the expression of CTGF in VSMCs and CFs affected by Ang II and advanced glycation end-products $^{[37,38]}$. As with CTGF, PAI-1 is also a potential pro-fibrotic factor and is involved in fibrosis in a variety of tissues, such as lung, heart, and liver ${ }^{[7]}$. Additionally, the expression of PAI-1 is also partially regulated by PPAR- $\gamma$ in Ang II-stimulated $\mathrm{CFs}^{[8]}$. In the present study, we demonstrated that in SHRs, the expression of CTGF and PAI-1 in left ventricle was dramatically elevated at the mRNA and protein levels, suggesting that CTGF and PAI-1 are partially involved in the progression of cardiac fibrosis induced by hypertension. The upregulation of PPAR- $\gamma$ activity by Cur caused a significant decrease in both the in vitro and in vivo expression of CTGF and PAI-1.

As Cur has a blood pressure-lowering effect, we determined whether the inhibitory effect of Cur on Ang II-induced expression of CTGF and PAI-1 is truly PPAR- $\gamma$-dependent, and not blood pressure- or hemodynamic change-dependent, by using CF primary cultures to evaluate the effect of Cur on Ang IIinduced expression of CTGF and PAI-1. Ang II elevated the expression of CTGF and PAI-1 at both mRNA and protein levels, and decreased the expression of PPAR- $\gamma$ and DNAbinding activity in CFs. Treatment with Cur increased the expression and DNA-binding activity of PPAR- $\gamma$ in a dosedependent manner. At the same time, Cur attenuated Ang II-induced expression of CTGF and PAI-1 at both the mRNA and protein levels in CFs: this effect was reversed by pretreatment with GW9662, a PPAR-ץ antagonist. These results demonstrate the direct cellular effect of Cur on Ang II-induced expression of pro-fibrotic factors, and indicate that the effect is partially PPAR- $\gamma$-dependent.
TGF- $\beta$ signaling plays an important role in the progression of cardiac fibrosis by modulating myofibroblast differentiation, enhancing ECM production, and suppressing the activity of $\mathrm{MMPs}^{[32]}$. Furthermore, some studies have demonstrated that Ang II upregulates TGF- $\beta$ mRNA and protein expression in $\mathrm{CF}^{[39]}$. Treatment with AT1 inhibitors markedly blocked Ang II-induced expression of TGF- $\beta$ in hypertrophied and infarcted hearts, suggesting that TGF- $\beta$ is a downstream target of Ang $\mathrm{II}^{[39]}$. It has been demonstrated that TGF- $\beta$ is a strong inducer of CTGF and PAI in animal model of cardiac remolding ${ }^{[33,40]}$. More importantly, previous studies have found that the expression TGF- $\beta$ is partially controlled by PPAR- $\gamma$, and some PPAR- $\gamma$-ligands markedly suppress TGF- $\beta$ mRNA and protein expression ${ }^{[8,14]}$. In addition, growing evidence has focused on the relationship between the pharmacological effect of Cur and TGF- $\beta$ signaling. It has been demonstrated that Cur significantly inhibits TGF- $\beta$ production and TGF- $\beta$ signaling in the setting of bile duct obstruction- and $\mathrm{CCl}_{4}$-induced hepatic injury, as well as in renal cells, and scleroderma fibroblasts, through a range of molecular mechanisms ${ }^{[41-45]}$. Smad2/3 is the primary downstream target of TGF- $\beta$, which is involved in the expression of CTGF and PAI and ECM production in different cell types and tissues and is a main target of Cur's pharmacological effect on TGF- $\beta$ signaling, a process that inhibits the proliferation of different cell types ${ }^{[46,47-49]}$. However, prior to this study, whether Cur inhibited Ang II-induced TGF- $\beta$ signaling, and whether PPAR- $\gamma$ was involved in this process, was unknown. We found that treating CFs with Ang II remarkably increased TGF- $\beta 1$ mRNA and protein expression, as well as the phosphorylation of Smad2/3. This effect was attenuated by pretreatment with Cur. Furthermore, the PPAR- $\gamma$ antagonist, GW9662, partially reversed the inhibitory effect of Cur on Ang II-induced expression of TGF- $\beta 1$ and phosphorylation of Smad2/3 in CFs. These results demonstrate that TGF- $\beta /$ Smad2/3 signaling may be involved in Cur's inhibitory effect on Ang II-induced CF proliferation, ECM production, and expression of PAI-1 and CTGF in a manner partially mediated by PPAR- $\gamma$.

Although our present study has demonstrated that Cur inhibits Ang II-induced expression of CTGF and PAI, as well as ECM production, these results may not clearly reflect whether Cur effectively suppresses Ang II-induced cardiac fibrosis in animal models. Therefore, we choose spontaneously hypertensive rats, which are characterized by a high concentration of Ang II, as the animal models in which to observe the inhibitory effect of Cur on the expression of profibrotic factors and ECM production caused by hypertension. Previous evidence has demonstrated that the expression of CTGF, PAI, and ECM is markedly elevated in SHRs compared with normal rats ${ }^{[37,50]}$. Recently, it has been shown that by suppressing ECM production and CF proliferation, Cur has the potential to prevent post-infarction cardiac remodeling ${ }^{[33]}$. This is the first study to demonstrate that Cur prevents the progression of cardiac fibrosis in SHRs, as indicated by a dosedependent decrease in CTGF, PAI, Col III, and FN production in the left ventricle. Furthermore, Cur also abrogates the 
elevated ratios of heart weight/body weight and left ventricle weight/body weight observed in SHRs. The expression of PPAR- $\gamma$ was dramatically lower in SHRs, a scenario associated with elevated blood pressure that was attenuated by pretreatment with Cur. However, the PPAR-y antagonist, GW9662, reversed the inhibitory effect of Cur on the progression of cardiac fibrosis in SHRs. These results clearly demonstrated that Cur suppressed cardiac fibrosis in SHRs, as indicated by inhibition of the production of CTGF, PAI-1, Col III, and FN in the left ventricle, as well as the decreases in the ratios of heart weight/body weight and left ventricle weight/body weight, event mediated in part by PPAR- $\gamma$. Cur decreased SBP in SHRs, and previous studies have also reported that Cur decreases the level of SBP in L-NAME-induced Wistar rats. Due to GW9662 (the PPAR-ץ antagonist) preventing decrease in SBP in SHRs, it is possible that the activity of PPAR-ץ was at least partly involved in this process. Growing evidence has demonstrated that PPAR-Y agonists such as rosiglitazone and pioglitazone effectively downregulate SBP in diabetic patients and animal models ${ }^{[51-54]}$. Additional research is needed to determine whether the anti-fibrotic effect of Cur is related to its inhibitory effect on blood pressure in SHRs.

Overall, the present study demonstrates that Cur inhibits Ang II-induced CTGF, PAI, and ECM production in CFs, and attenuates cardiac fibrosis in SHRs, and its effects are partially dependent on cross-talk between PPAR- $\gamma$ and TGF- $\beta$ / Smad2/3 signaling. These results suggest a possible novel mechanism that acts to prevent cardiac fibrosis caused by hypertension.

\section{Acknowledgements}

This study was supported by the Youth Foundation of The First Affiliated Hospital of Zhengzhou University [YFFAHZZ 2013020105 to Zhe MENG].

\section{Author contribution}

Most of the experiments were conducted by Zhe MENG and Xin-hui YU, who also wrote this paper. Jun CHEN also conducted some of the experiments. Ling LI and Sheng LI designed the experiments together.

\section{References}

1 Leask A. Potential therapeutic targets for cardiac fibrosis: TGFbeta, angiotensin, endothelin, CCN2, and PDGF, partners in fibroblast activation. Circ Res 2010; 106: 1675-80.

2 Krenning G, Zeisberg EM, Kalluri R. The origin of fibroblasts and mechanism of cardiac fibrosis. J Cell Physiol 2010; 225: 631-7.

3 Shimosawa T. Salt, the renin-angiotensin-aldosterone system and resistant hypertension. Hypertens Res 2013; 36: 657-60.

4 Askari AT, Shishehbor MH, Kaminski MA, Riley MJ, Hsu A, Lincoff AM. The association between early ventricular arrhythmias, reninangiotensin-aldosterone system antagonism, and mortality in patients with ST-segment-elevation myocardial infarction: Insights from Global Use of Strategies to Open coronary arteries (GUSTO) V. Am Heart J 2009; 158: 238-43.

5 Siddesha JM, Valente AJ, Sakamuri SS, Yoshida T, Gardner JD, Somanna $\mathrm{N}$, et al. Angiotensin II stimulates cardiac fibroblast migration via the differential regulation of matrixins and RECK. J Mol Cell Cardiol 2013; 65: 9-18.

6 de Cavanagh EM, Ferder M, Inserra F, Ferder L. Angiotensin II, mitochondria, cytoskeletal, and extracellular matrix connections: an integrating viewpoint. Am J Physiol Heart Circ Physiol 2009; 296: H550-8.

7 Ghosh AK, Vaughan DE. PAl-1 in tissue fibrosis. J Cell Physiol 2012; 227: 493-507.

8 Hao GH, Niu XL, Gao DF, Wei J, Wang NP. Agonists at PPAR-gamma suppress angiotensin II-induced production of plasminogen activator inhibitor-1 and extracellular matrix in rat cardiac fibroblasts. $\mathrm{Br} \mathrm{J}$ Pharmacol 2008; 153: 1409-19.

9 Weisberg AD, Albornoz F, Griffin JP, Crandall DL, Elokdah H, Fogo $A B$, et al. Pharmacological inhibition and genetic deficiency of plasminogen activator inhibitor-1 attenuates angiotensin II/saltinduced aortic remodeling. Arterioscler Thromb Vasc Biol 2005; 25: 365-71.

10 Dean RG, Balding LC, Candido R, Burns WC, Cao Z, Twigg SM, et al. Connective tissue growth factor and cardiac fibrosis after myocardial infarction. J Histochem Cytochem 2005; 53: 1245-56.

11 Oemar BS, Werner A, Garnier JM, Do DD, Godoy N, Nauck M, et al. Human connective tissue growth factor is expressed in advanced atherosclerotic lesions. Circulation 1997; 95: 831-9.

12 Jaffa AA, Usinger WR, McHenry MB, Jaffa MA, Lipstiz SR, Lackland $D$, et al. Connective tissue growth factor and susceptibility to renal and vascular disease risk in type 1 diabetes. J Clin Endocrinol Metab 2008; 93: 1893-900.

13 Liu X, Gai Y, Liu F, Gao W, Zhang Y, Xu M, et al. Trimetazidine inhibits pressure overload-induced cardiac fibrosis through NADPH oxidaseROS-CTGF pathway. Cardiovasc Res 2010; 88: 150-8.

14 Gao DF, Niu XL, Hao GH, Peng N, Wei J, Ning N, et al. Rosiglitazone inhibits angiotensin II-induced CTGF expression in vascular smooth muscle cells-role of PPAR-gamma in vascular fibrosis. Biochem Pharmacol 2007; 73: 185-97.

15 Ahmed MS, Oie E, Vinge LE, Yndestad A, Oystein AG, Andersson Y, et al. Connective tissue growth factor - a novel mediator of angiotensin II-stimulated cardiac fibroblast activation in heart failure in rats. J Mol Cell Cardiol 2004; 36: 393-404.

16 Derosa G, Maffioli P. Peroxisome proliferator-activated receptorgamma (PPAR-gamma) agonists on glycemic control, lipid profile and cardiovascular risk. Curr Mol Pharmacol 2012; 5: 272-81.

17 Ji Y, Liu J, Wang Z, Liu N, Gou W. PPARgamma agonist, rosiglitazone, regulates angiotensin II-induced vascular inflammation through the TLR4-dependent signaling pathway. Lab Invest 2009; 89: 887-902.

18 Goyal S, Arora S, Bhatt TK, Das P, Sharma A, Kumari S, et al. Modulation of PPAR-gamma by telmisartan protects the heart against myocardial infarction in experimental diabetes. Chem Biol Interact 2010; 185: 271-80.

19 Goel A, Kunnumakkara AB, Aggarwal BB. Curcumin as "Curecumin": from kitchen to clinic. Biochem Pharmacol 2008; 75: 787-809.

20 Meng Z, Yan C, Deng Q, Gao DF, Niu XL. Curcumin inhibits LPS-induced inflammation in rat vascular smooth muscle cells in vitro via ROSrelative TLR4-MAPK/NF-KB pathways. Acta Pharmacol Sin 2013; 34: 901-11.

21 Aggarwal BB, Sung B. Pharmacological basis for the role of curcumin in chronic diseases: an age-old spice with modern targets. Trends Pharmacol Sci 2009; 30: 85-94.

22 Bar-Sela G, Epelbaum R, Schaffer M. Curcumin as an anti-cancer agent: review of the gap between basic and clinical applications. Curr Med Chem 2010; 17: 190-7.

23 Lin JK. Molecular targets of curcumin. Adv Exp Med Biol 2007; 595: 
227-43.

24 Hlavackova L, Janegova A, Ulicna O, Janega P, Cerna A, Babal P. Spice up the hypertension diet - curcumin and piperine prevent remodeling of aorta in experimental L-NAME induced hypertension. Nutr Metab (Lond) 2011; 8: 72.

25 Coban D, Milenkovic D, Chanet A, Khallou-Laschet J, Sabbe L, Palagani $A$, et al. Dietary curcumin inhibits atherosclerosis by affecting the expression of genes involved in leukocyte adhesion and transendothelial migration. Mol Nutr Food Res 2012; 56: 1270-81.

26 Yu W, Wu J, Cai F, Xiang J, Zha W, Fan D, et al. Curcumin alleviates diabetic cardiomyopathy in experimental diabetic rats. PLoS One 2012; 7: e52013.

27 Liu ZJ, Liu W, Liu L, Xiao C, Wang Y, Jiao JS. Curcumin protects neuron against cerebral ischemia-induced inflammation through improving PPAR-gamma function. Evid Based Complement Alternat Med 2013; 2013: 470975.

28 Lin J, Tang Y, Kang Q, Feng Y, Chen A. Curcumin inhibits gene expression of receptor for advanced glycation end-products (RAGE) in hepatic stellate cells in vitro by elevating PPARgamma activity and attenuating oxidative stress. Br J Pharmacol 2012; 166: 2212-27.

29 Kukongviriyapan U, Pannangpetch P, Kukongviriyapan V, Donpunha W, Sompamit K, Surawattanawan P. Curcumin protects against cadmiuminduced vascular dysfunction, hypertension and tissue cadmium accumulation in mice. Nutrients 2014; (6): 1194-208.

30 Kim S, Ohta K, Hamaguchi A, Yukimura T, Miura K, Iwao H. Angiotensin II induces cardiac phenotypic modulation and remodeling in vivo in rats. Hypertension 1995; 25: 1252-9.

31 Shiomi T, Tsutsui H, Hayashidani S, Suematsu N, Ikeuchi M, Wen J, et al. Pioglitazone, a peroxisome proliferator-activated receptor-gamma agonist, attenuates left ventricular remodeling and failure after experimental myocardial infarction. Circulation 2002; 106: 3126-32.

32 Yao QH, Wang DQ, Cui CC, Yuan ZY, Chen SB, Yao XW, et al. Curcumin ameliorates left ventricular function in rabbits with pressure overload: inhibition of the remodeling of the left ventricular collagen network associated with suppression of myocardial tumor necrosis factoralpha and matrix metalloproteinase-2 expression. Biol Pharm Bull 2004; 27: 198-202.

33 Liu JJ, Li DL, Zhou J, Sun L, Zhao M, Kong SS, et al. Acetylcholine prevents angiotensin II-induced oxidative stress and apoptosis in H9c2 cells. Apoptosis 2011; 16: 94-103.

34 Wang Q, Usinger W, Nichols B, Gray J, Xu L, Seeley TW, et al. Cooperative interaction of CTGF and TGF-beta in animal models of fibrotic disease. Fibrogenesis Tissue Repair 2011; 4: 4.

35 Ko WC, Hong CY, Hou SM, Lin CH, Ong ET, Lee CF, et al. Elevated expression of connective tissue growth factor in human atrial fibrillation and angiotensin II-treated cardiomyocytes. Circ J 2011; 75 : 1592-600.

36 Cai Y, Yu SS, Chen TT, Gao S, Geng B, Yu Y, et al. EGCG inhibits CTGF expression via blocking NF-kappaB activation in cardiac fibroblast. Phytomedicine 2013; 20: 106-13.

37 Gao D, Ning N, Hao G, Niu X. Pioglitazone attenuates vascular fibrosis in spontaneously hypertensive rats. PPAR Res 2012; 2012: 856426.

38 Li J, Liu NF, Wei Q. Effect of rosiglitazone on cardiac fibroblast proliferation, nitric oxide production and connective tissue growth factor expression induced by advanced glycation end-products. J Int Med Res 2008; 36: 329-35.

39 Gray MO, Long CS, Kalinyak JE, Li HT, Karliner JS. Angiotensin II stimulates cardiac myocyte hypertrophy via paracrine release of TGFbeta 1 and endothelin-1 from fibroblasts. Cardiovasc Res 1998; 40: 352-63.

40 Byrne CD, Wareham NJ, Martensz ND, Humphries SE, Metcalfe JC, Grainger DJ. Increased PAl activity and PAI-1 antigen occurring with an oral fat load: associations with PAl-1 genotype and plasma active TGF-beta levels. Atherosclerosis 1998; 140: 45-53.

41 Baghdasaryan A, Claudel T, Kosters A, Gumhold J, Silbert D, Thuringer $A$, et al. Curcumin improves sclerosing cholangitis in Mdr2-/- mice by inhibition of cholangiocyte inflammatory response and portal myofibroblast proliferation. Gut 2010; 59: 521-30.

42 Fu Y, Zheng S, Lin J, Ryerse J, Chen A. Curcumin protects the rat liver from $\mathrm{CCl}_{4}$-caused injury and fibrogenesis by attenuating oxidative stress and suppressing inflammation. Mol Pharmacol 2008; 73 : 399-409.

43 Reyes-Gordillo K, Segovia J, Shibayama M, Tsutsumi V, Vergara P, Moreno MG, et al. Curcumin prevents and reverses cirrhosis induced by bile duct obstruction or $\mathrm{CCl}_{4}$ in rats: role of TGF-beta modulation and oxidative stress. Fundam Clin Pharmacol 2008; 22: 417-27.

44 Li R, Wang Y, Liu Y, Chen Q, Fu W, Wang H, et al. Curcumin inhibits transforming growth factor-beta1-induced EMT via PPARgamma pathway, not Smad pathway in renal tubular epithelial cells. PLoS One 2013; 8: e58848.

45 Song K, Peng S, Sun Z, Li H, Yang R. Curcumin suppresses TGF-beta signaling by inhibition of TGIF degradation in scleroderma fibroblasts. Biochem Biophys Res Commun 2011; 411: 821-5.

46 Dobaczewski M, Chen W, Frangogiannis NG. Transforming growth factor (TGF)-beta signaling in cardiac remodeling. J Mol Cell Cardiol 2011; 51: 600-6.

$47 \mathrm{Hu} \mathrm{Y,} \mathrm{Liang} \mathrm{H,} \mathrm{Du} \mathrm{Y,} \mathrm{Zhu} \mathrm{Y,} \mathrm{Wang} \mathrm{X.} \mathrm{Curcumin} \mathrm{inhibits} \mathrm{transforming}$ growth factor-beta activity via inhibition of Smad signaling in HK-2 cells. Am J Nephrol 2010; 31: 332-41.

48 Ryu HW, Kim SP, Lee KS, Cho JW. Curcumin induced decreased expression of type I collagen in human skin fibroblast through downregulation of Smad2/3 expressions. Korean J Dermatol 2012; 50: $1-7$.

49 Song K, Peng S, Sun Z, Li H, Yang R. Curcumin suppresses TGF-beta signaling by inhibition of TGIF degradation in scleroderma fibroblasts. Biochem Biophys Res Commun 2011; 411: 821-5.

50 Cheng X, Zhou Q, Lin S, Wu R. Fosinopril and valsartan intervention in gene expression of Klotho, MMP-9, TIMP-1, and PAI-1 in the kidney of spontaneously hypertensive rats. J Central South Univ Med Sci 2010; 35: 1048-56.

51 Sartori-Valinotti JC, Venegas-Pont MR, Lamarca BB, Romero DG, Yanes LL, Racusen LC, et al. Rosiglitazone reduces blood pressure in female Dahl salt-sensitive rats. Steroids 2010; 75: 794-9.

52 Hamblin M, Chang L, Zhang J, Chen YE. The role of peroxisome proliferator-activated receptor gamma in blood pressure regulation. Curr Hypertens Rep 2009; 11: 239-45.

53 Schneider F, Vossler S, Franke S, Bar F, Konrad T. Impact of insulin sensitivity treatment with pioglitazone on endothelial function in nondiabetic patients with arterial hypertension. Int J Clin Pharmacol Ther 2009; 47: 311-20.

54 Tian YQ, Li SS, Su XD, Zhang GZ, Zhao JJ, Li GW, et al. Effects of pioglitazone on high-fat-diet-induced ventricular remodeling and dysfunction in rats. J Cardiovasc Pharmacol Ther 2012; 17: 223-8. 